\title{
Redende Einbände
}

Ein Einband ist Teil der ästhetischen Gesamtkomposition eines Buches, hat aber je nach Gestaltung auch eine ästhetische und inhaltliche Eigenaussage. Das Phänomen eines "redenden Einbandes" (reliure parlante) stellt eine Sonderform der Wechselwirkung zwischen Buchblock und Einband dar. Mit "redenden Einbänden" sind nicht die zahlreichen Fälle gemeint, in denen sich das "Reden" auf das Buch überhaupt bezieht oder sich eher zufällig ein Bezug auf den Inhalt des jeweiligen Buches ergibt. Der Aussagegehalt der ästhetischen Gestaltung eines Einbandes ist in der Regel unabhängig vom Buchinhalt, weckt allerdings Interesse für das Buch. Die Tatsache, nicht die Motivik der Einbandverzierung führt zum Buch hin.

Historische Aussagekraft entwickelt ein Einband durch Provenienzhinweise wie Wappen-Supralibros oder aufgeprägte Besitzernamen bzw. Namensinitialen und Motti. Zweck und Inhalt kommen zusammen bei mittelalterlichen Bibelhandschriften mit Silber- oder Goldschmiedearbeiten, Elfenbein und Edelsteinen. Ein in dieser Weise gestaltetes Buch ist quasi ein liturgisches Gerät und weist über sich hinaus auf den himmlisch-ewigen Gegenstand des Gottesdienstes, macht die Gegenwart Gottes im Hier und Jetzt manifest. ${ }^{1}$

Seit der Renaissance kommt es zu einer Akzentverschiebung zur subjektiven Seite im Verhältnis zwischen Mensch und Buch. Dem Einband für sich genommen kommt dabei eine größere Bedeutung $z_{\text {zu. }}{ }^{2}$ Der Einband wird sozusagen als Schauseite des Buches zum primären Artikulationsmittel eines geistigen Programms nicht nur des Büchersammlers, sondern einer ganzen Epoche. Gelegentliche Neubindungen im Stil der Zeit sind Indizien für einen Richtungswechsel des gesellschaftlichen Selbstverständnisses. Der Einband wirbt für die Anliegen eines Kunststils, aber epochenübergreifend auch für Bibliophilie und das Buch allgemein. Ilse Schunke wertet den seit der industriellen Produktion von Einbänden häufiger werdenden inhaltlichen Bezug ab, weil die Kunst dann nicht mehr Selbstzweck, sondern apologetisches Mittel zum Zweck der Veranschaulichung bestimmter Inhalte ist. ${ }^{3}$
Demgegenüber spricht manches dafür, die Akzentuierung der objektiven Seite, also der Beziehung zwischen Einband und Buch, positiver zu bewerten. Das Beispiel der über sich hinausweisenden mittelalterlichen Prachteinbände zeigt, dass die inhaltliche Zweckdienlichkeit des Einbandes nicht der Entfaltung künstlerischer Kreativität entgegenstehen muss. Vielmehr kann gerade die Hochschätzung eines Inhalts dazu motivieren, eine dem Gegenstand entsprechende ästhetische Gestalt zu entwickeln. Auch das kommerzielle Interesse schließt nicht inhaltliche Festlegungen des Einbandes aus. Ein Buch wird primär wegen seines Inhalts gekauft. Ein inhaltlich definierter Einband als Ouvertüre fördert die Kaufentscheidung. Freilich lohnt sich der Aufwand einer auf ein Einzelwerk bezogenen Einbandgestaltung mehr, wenn wie bei Verlagseinbänden eine ganze Auflage diesen individuell konzipierten Einband erhält. Künstlerische Individualität richtet sich vor allem in Gotik und Renaissance auf die Konstruktion eines dann vielfach verwendeten Werkzeuges aus. Sie kommt bei redenden Einbänden an anderer Stelle, nämlich bei der Art und Weise der Einbandoberfläche als Ganzes, zum Ausdruck, wobei allgemein gebräuchliche Werkzeuge genutzt werden.

Geht die Bewegungsrichtung bei nicht-redenden Handeinbänden vom bibliophilen Sammler zum Buch, so geht sie bei redenden Einbänden vom Buchinhalt über den Einband zum Käufer. Interesse an einem bestimmten Buchinhalt kann bei überzeugender Gesamtkomposition eine Liebe zum Buch überhaupt wecken, aus reinen Lesern womöglich begeisterte Bibliophile machen. Wie die Buchillustration wirkt eine auf den Buchinhalt bezogene Motivik der Einbandgestaltung elementarisierend. Komplexe Inhalte werden auf entscheidende Einzelaspekte zugespitzt, auch vereinfacht und stilisiert. Bildliche Konkretion wirkt anregend, ruft Assoziationen hervor, ermöglicht ganzheitliches Lernen.

Anhand mehrerer Beispiele aus dem Bestand der Württembergischen Landesbibliothek Stuttgart werden Typen redender Einbände aufgezeigt. 


\section{Technische Komposition}

Eine Sonderform redender Einbände liegt vor, wenn sich die inhaltliche Aussage auf die technische Methode und Komposition des Einbandes bezieht. Mehrfachbände gehören zu den Kuriositäten der Einbandkunst. Bei einer als Drillingsband gestalteten Bibel befindet sich das Neue Testament in der Mitte und wird umrahmt von den beiden Hälften des Alten Testaments. Zudem muss man, um das Neue Testament zu lesen, das Buch um $180^{\circ}$ drehen (Signatur WLB Stuttgart: B deutsch 165401 / ES 37). Das sagt in Kombination mit dem enthaltenen Text aus: Altes und Neues Testament sind in einem heilsgeschichtlichen Spannungsgefälle aufeinander bezogen. Das Alte Testament zielt auf das Neue ab, führt allerdings nicht bruchlos darauf hin.

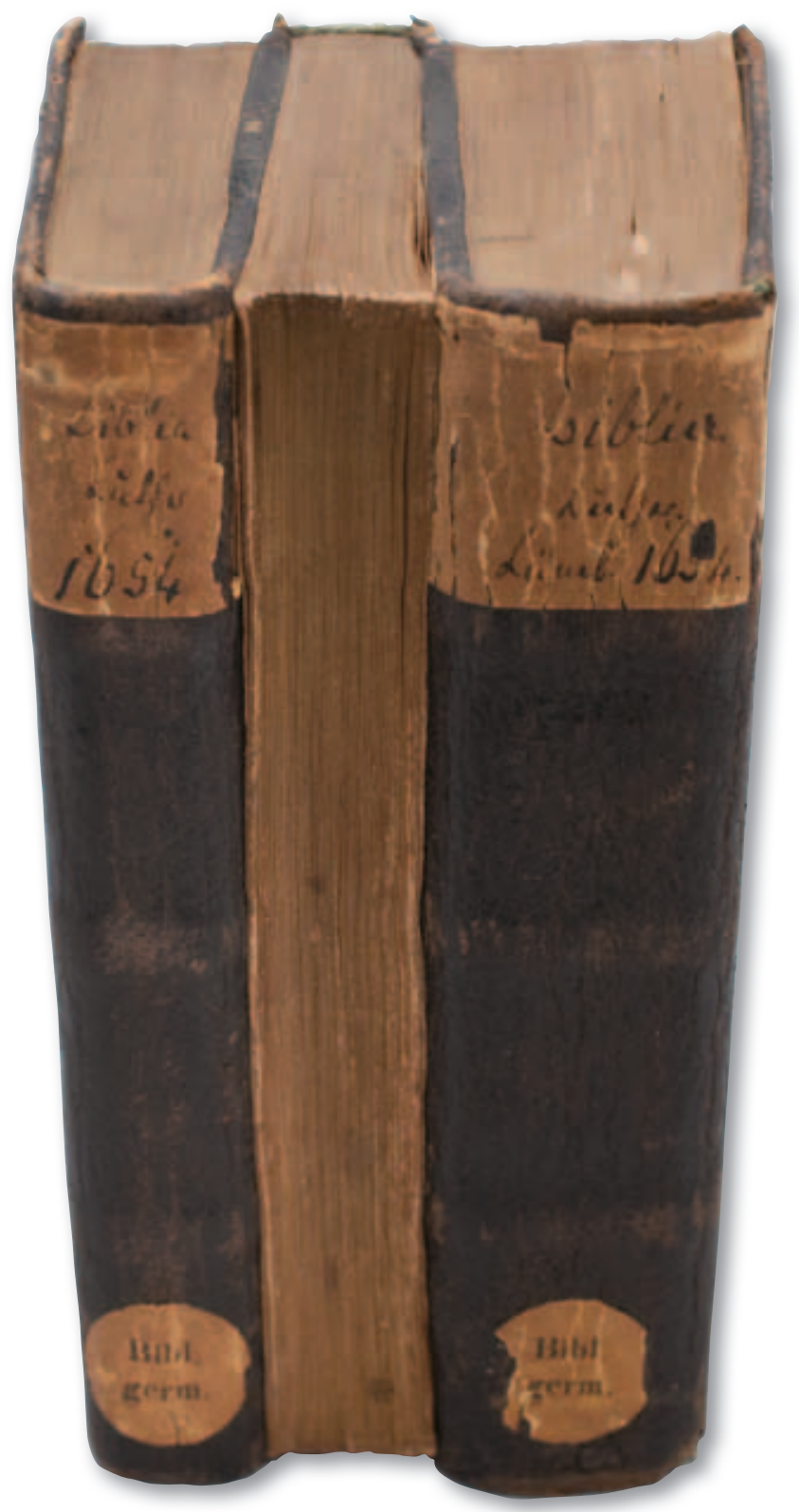

B deutsch 165401 / ES 37

\section{Kombination abstrakter und figürlicher Motive}

Bei einer 1720 gedruckten Prachtausgabe der Weimarer Kurfürstenbibel des Nürnberger EndterVerlags (Signatur WLB Stuttgart: Bb graph. 1720 01) liegt eine Kombination abstrakter und figürlicher Motive barocker Einbandgestaltung vor. Bandwerkornamente, Fächermuster, Blatt- und Rankenstempel in Goldprägung und feiner Punktierung unterstreichen mit ihrem Detailreichtum und ihrer künstlerischen Perfektion die Bedeutung des Buches. Objektiv ist dieses Buch wichtig und dekorationswürdig als Bibel und im wahrscheinlichen Zweck als Altar- und Gemeindebibel. Subjektiv ist dieses Buch bedeutsam für seinen Besitzer, der die Einbandgestaltung in Auftrag gibt. Hinweis auf den Inhalt geben die fein strukturierten Metallauflagen. Auf bronzenen Halbreliefs sieht man Mose mit den Gesetzestafeln bzw. Christus als Weltheiland mit der Weltkugel, verteilt auf Vorder- bzw. Hinterdeckel. Dies deutet in plastischer Weise auf den Zusammenhang von Altem und Neuem Bund hin. Die Schließen enden in zwei ebenfalls bronzenen Halbreliefs mit David bzw. Aaron. Sie haben als Vertreter von weltlicher bzw. geistlicher Macht, als König bzw. Priester die Ausrichtung auf Gott gemeinsam. Ihr vorbildliches Verhalten verpflichtet kirchliche wie weltliche Amtsträger, als Förderer des Unternehmens Bibeldruck und Bibelverbreitung aktiv zu werden. Redend ist der Einband insofern, als er herausragende biblische Figuren künstlerisch zitiert, biblische Inhalte in den mit ihnen verbundenen Konnotationen konzentriert und den konkret-geschichtlichen Gehalt der Offenbarung Gottes vor Augen führt. Noch eindeutiger als abstrakte und figürliche Verzierung sind direkte Zitate aus der Bibel als Schriftdekor. Dabei handelt es sich um solche Verse aus den Psalmen, die die Bedeutung des Wortes Gottes herausstellen (Psalm 119,18; Psalm 84,2). Das Zueinander von Bild- und Schriftzitat kennzeichnet auch die Struktur der ganzseitigen Kupferstich-lllustrationen, mit denen diese Bibel ausgestattet ist. Bilder gewinnen an Eindeutigkeit, Texte an Anschaulichkeit. Den feierlichen Charakter der Bibel unterstreicht auch das Brokatpapier als Material für Spiegel und Vorsatz sowie der ziselierte Goldschnitt. Dabei handelt es sich allerdings nur um zusätzliche Dekorationselemente, denen keine eigenständige, sondern nur eine verstärkende Redekraft zukommt. 


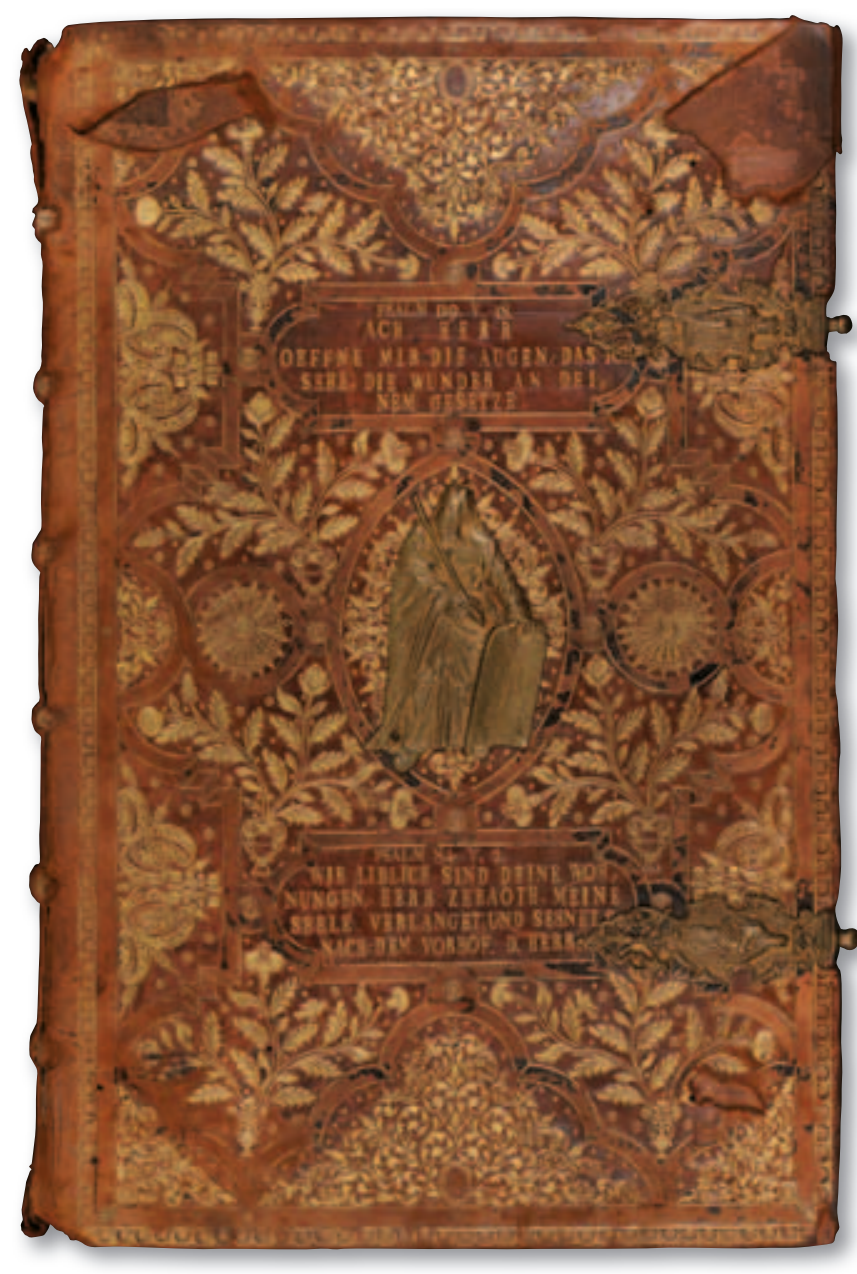

Bb graph. 172001

\section{Symbolik}

Die Holzstiche von Gustave Doré (1832-1883) zu biblischen Szenen werden in unterschiedlichen Kontexten nachgedruckt. Eindeutigkeit entsteht erst durch die Verwendung einer bestimmten Übersetzung, durch Typographie, Titelblatt und Einband. Eine jüdische, deutsche Ausgabe des Alten Testaments mit Doré-Illustrationen aus dem Jahr 1874 (Signatur WLB Stuttgart: Bb graph. 1874 $01)$ ist bereits an der Gestaltung ihres Verlagseinbandes zu erkennen. Die Titelprägung „Die Heilige Schrift der Israeliten" spricht aus, was bereits durch die Symbolik der Dekorationselemente angedeutet wird. In einem nach unten abgeschnittenen Medaillon erkennt man die Gesetzestafeln mit hebräischen Schriftzeichen. Kleine Eckmedaillons deuten den Übergang von einem am Opferkult im Tempel orientierten Judentum zur stärkeren Ausrichtung an der verbalen Thora im Diasporajudentum an. Man sieht die Bundeslade, einen Räucherbehälter, den Siebenarmigen Leuchter und einen Thoraschrein. Die Randleiste besteht aus repetierenden Sternen mit reliefartigem Rahmen in Form des Davidsterns. Im Zusammenhang des europäischen Judentums konnte lange Zeit die Kenntnis des Symbolgehaltes vorausgesetzt werden. So sind weitergehende Erläuterungen kaum notwendig, um Assoziationen zum Gegenstand des gebundenen Buches hervorzurufen. Das gilt in ähnlicher Weise für christliche Symbole wie Kreuz, Chi-Rho oder (mit Einschränkungen) Fisch, Evangelistensymbole.

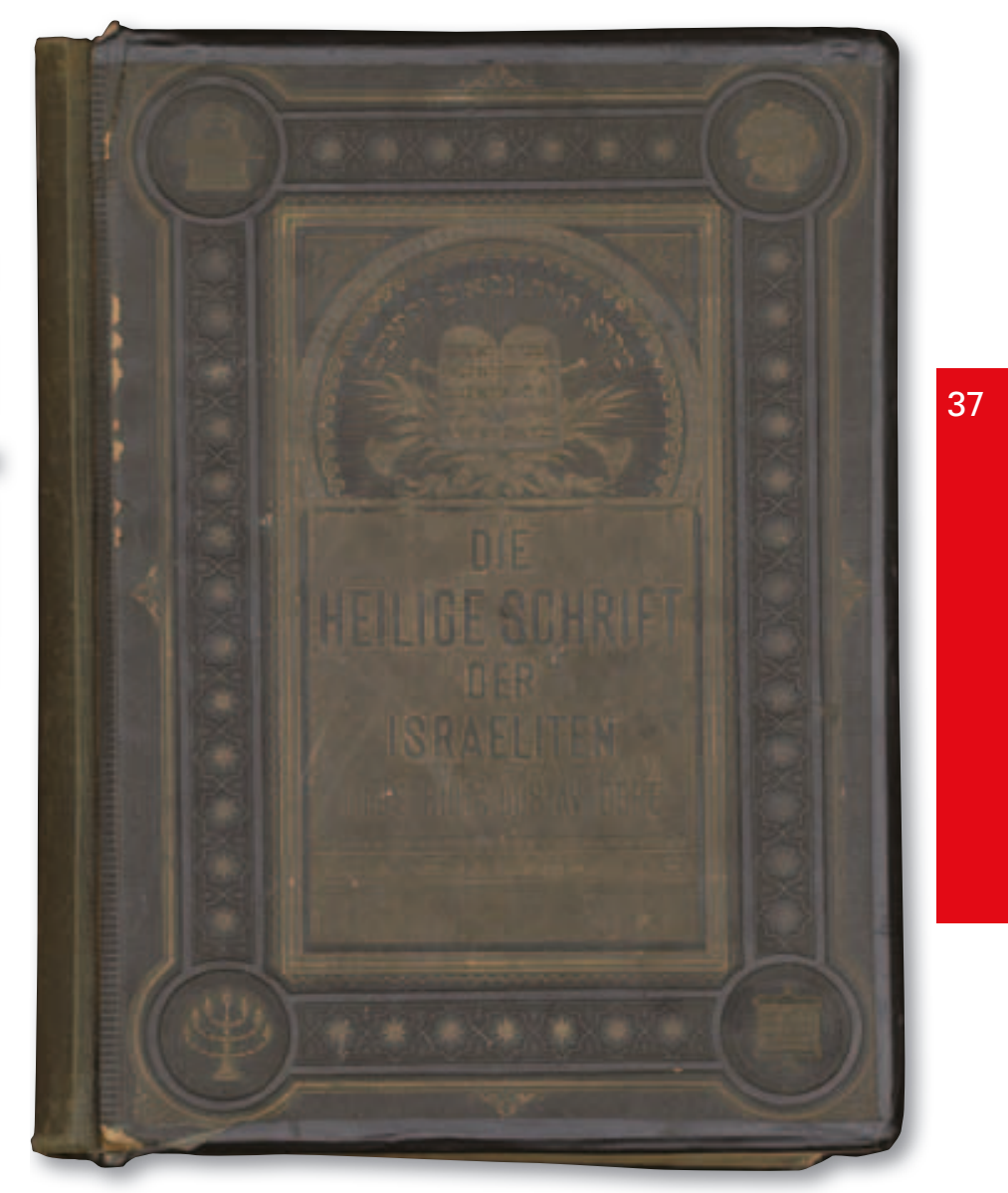

Bb graph. 187401

\section{Symbol und Text}

Die Kombination von Symbol und Textzitaten begegnet an einem von Gotthilf Kurz (1923-2010) angefertigten Handeinband um eine 1867 in Stuttgart gedruckte Lutherbibel mit braunem Schweinsledereinband (Signatur WLB Stuttgart: A 35/1).

Durch Blindprägung wird ein mit Linien bezeichnetes Kreuz mit einer stilisierten Blüte in seiner Mitte an den Deckeln angebracht. Die Linien werden über den Rücken fortgeführt und bilden auch dort ein Kreuzmotiv. Auf dem Rücken ist das Textelement "Gottes Wort" in Gestalt zweier Schriftbänder in das Kreuz verwoben. Flächen- und Schriftdekor sind geschickt miteinander verknüpft. Die Textelemente stellen nicht nur einen Zusatz zur 
Vereindeutigung dar. Zitate aus den Psalmen (vor allem Psalm 118 und Psalm 19) in Kapitalschrift bilden die Randleiste. Satzteile und ganze Sätze werden durch kleine Kreuze abgegrenzt.

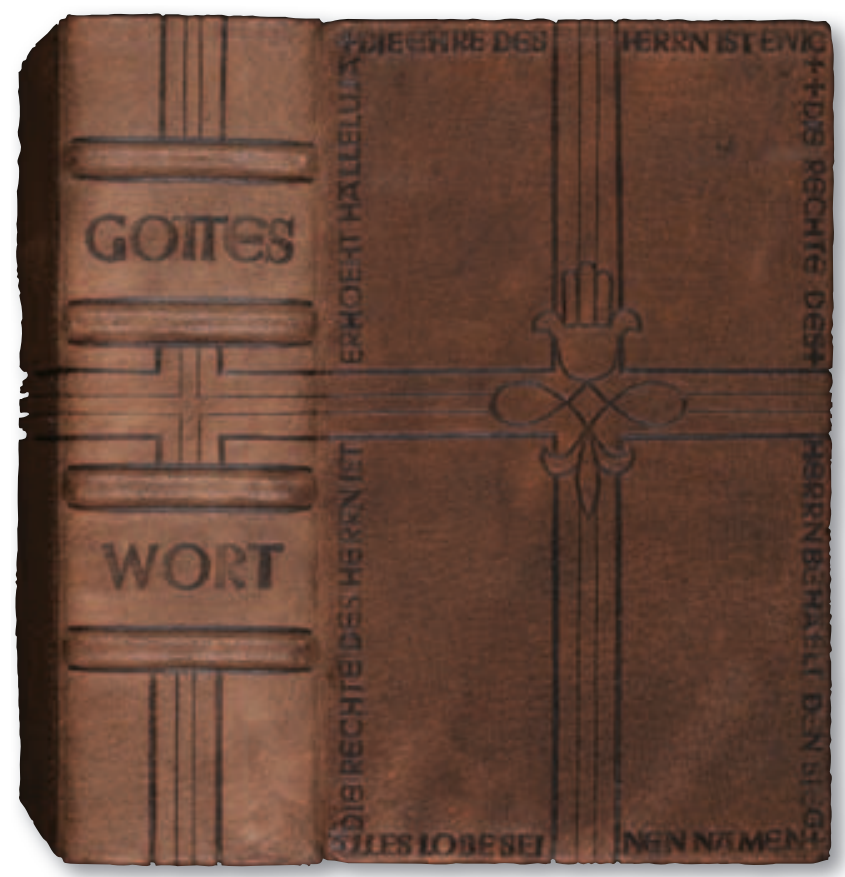

A 35/1

\section{Bezugsmaterialien}

Symbolik entsteht nicht nur durch bestimmte Formen, sondern auch durch die Wahl von Bezugsmaterialien. Das gilt etwa für eine 1837 gedruckte Karlsruher Bibel (Signatur WLB Stuttgart: Ba graph. 183701 / ES XIII). Zwar deuten auch hier ein in Silberschmiedearbeit gefertigtes Kreuz als zentrales Motiv sowie Putten als Eckstücke auf dem Vorderdeckel auf den transzendenten Buchinhalt hin. Jedoch wird die Überzeugungskraft dieser plastischen Symbole verstärkt durch den blauen Samt als Bezugsstoff. Samt findet vor allem Verwendung auf Einbänden aus dem Besitz von Mitgliedern regierender Fürstenhäuser. ${ }^{4}$ In diesem Fall handelt es sich mit großer Wahrscheinlichkeit um einen Angehörigen des Hauses Hannover, dem - wie handschriftlich vermerkt - die Bibel 1838 zur Konfirmation überreicht wird. Himmlische und irdische Herrschaft, Religion und Politik werden - ähnlich wie bei David und Aaron - in ihrem Wechselbezug, in Begründung wie Begrenzung aufgezeigt.

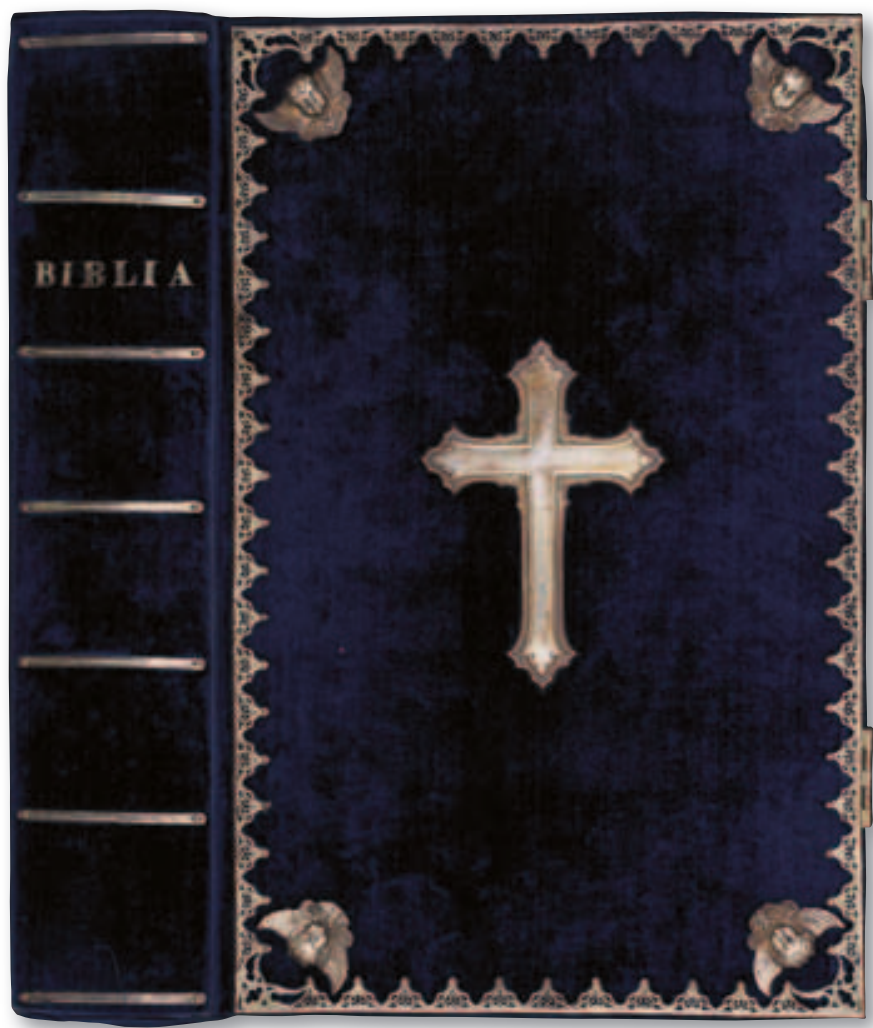

Ba graph. 183701 / ES XIII

\section{Konkret-stilisierte Motive mit Verallgemeinerungstendenz}

Eine andere Gruppe redender Einbände liegt dort vor, wo konkrete Motive ohne tiefergehenden Symbolgehalt aus dem Buchinhalt übernommen werden. Alltägliches, Diesseitiges wird meist in vereinfachter, stilisierter Form wiedergegeben.

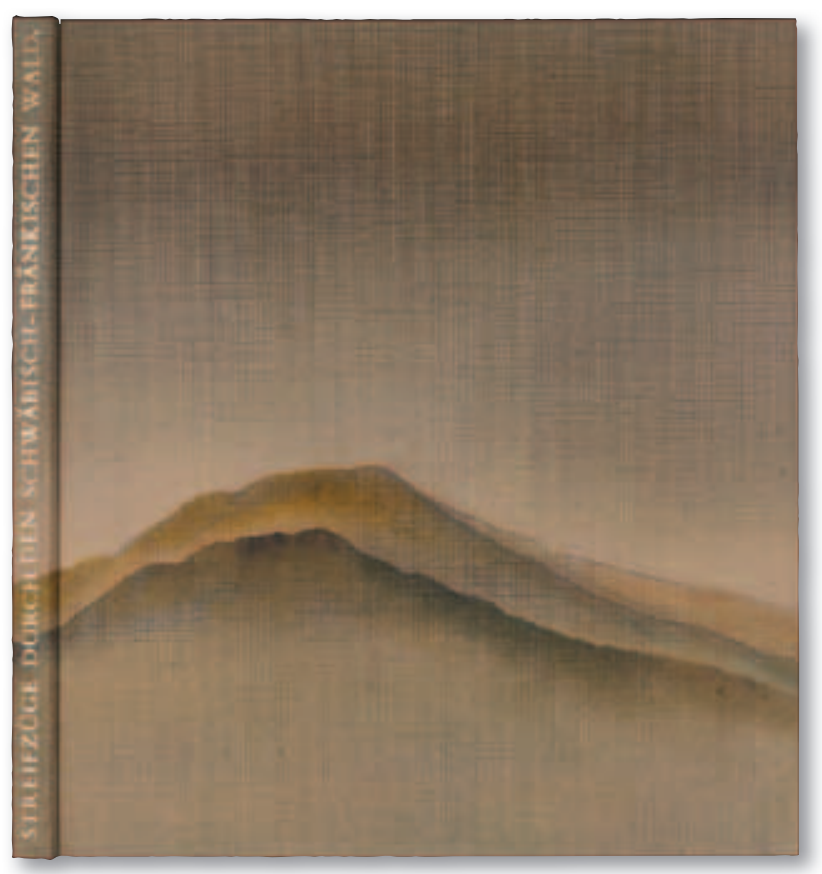

A 35/134

(4) So findet Samt auch Verwendung auf Büchern aus dem Besitz der russischen Frauen am württembergischen Königshof im 19. Jahrhundert. Z.B. Signatur WLB Stuttgart: W.G.oct. 608b; Bb kirchenslaw. 1762 01; Bb kirchenslaw. 1803 01; HB XV 83. 
Die Präzision von Buchinhalt und Dekorationsmotiv weichen in der ersten Teilgruppe mit konkreten Motiven stark ab. So malt Gotthilf Kurz eine Landschaftsszenerie auf den Pappband mit Gewebestruktur um einen 1987 erschienenen Bildband über den Schwäbisch-Fränkischen Wald (Signatur WLB Stuttgart: A 35/134). Spezifika gerade dieses Waldes können kaum mit den Mitteln der Einbandkunst angedeutet werden. Gezeigt wird vielmehr eine Waldlandschaft allgemein.

\section{Konkret-stilisierte Motive mit exakter Korrespondenzfunktion}

Anders ist es bei einer bibliophilen, im Leipziger Insel-Verlag 1905 herausgebrachten Ausgabe von Clemens Brentanos "Gockel, Hinkel, Gackeleien" (Signatur WLB Stuttgart: Sammlung Borst: SB 1856(3)). Es geht um Hühner allgemein, und so korrespondiert dem Titel bzw. Buchinhalt in präziser Weise die stilisierte Silhouette eines Hahns in einem geprägten Einbandmedaillon.

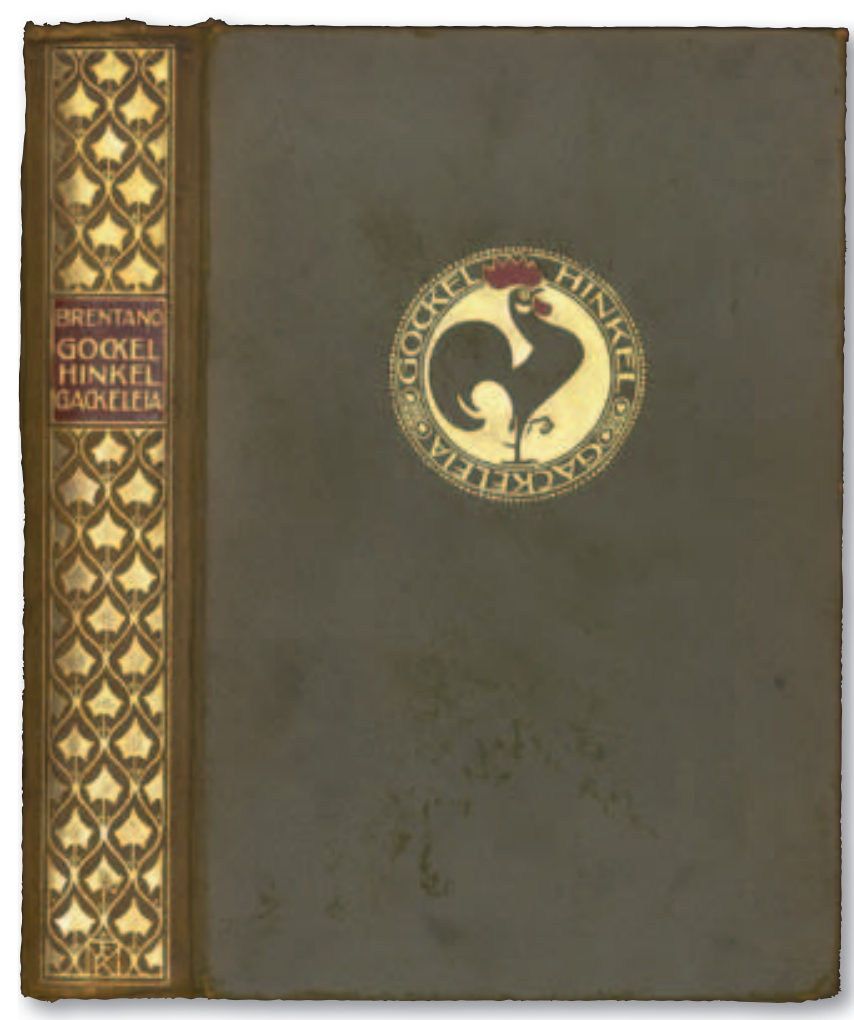

SB 1856(3)

\section{Naturalistisch-figürliche Motive}

Das Interesse des Historismus an konkreten Bezugspunkten aus der Geschichte führt bei Buchthemen mit historischen Bezügen zur Verwendung naturalistisch-figürlicher Motive auf dem Einband. Ein Höchstmaß zeitgeschichtlicher Kontextualisierung ist erreicht, wenn z.B. bei einer Abhandlung über die Geschichte des Berliner Theaters mit dem Titel "Unter zwei Königen“ (Signatur WLB Stuttgart: 62/90004 / ES 44) auf dem Vorderdeckel gemalte Porträts der preußischen Könige Friedrich Wilhelm IV. und Wilhelm I. erscheinen. Redend ist der Einband hier ganz unmittelbar, weil die Motivik historische Fakten ohne Interpretationsbedarf nachzeichnet und damit erläuternde wie zum Buchinhalt direkt hinführende Informationen in visueller Gestalt gibt.

Die Dokumentation von Einbänden trägt bei Wahrnehmung ihrer Redekraft erheblich dazu bei, Buchinhalte besser zu verstehen und Profil wie Intention von Bibliotheken besser einzuordnen.

Christian Herrmann

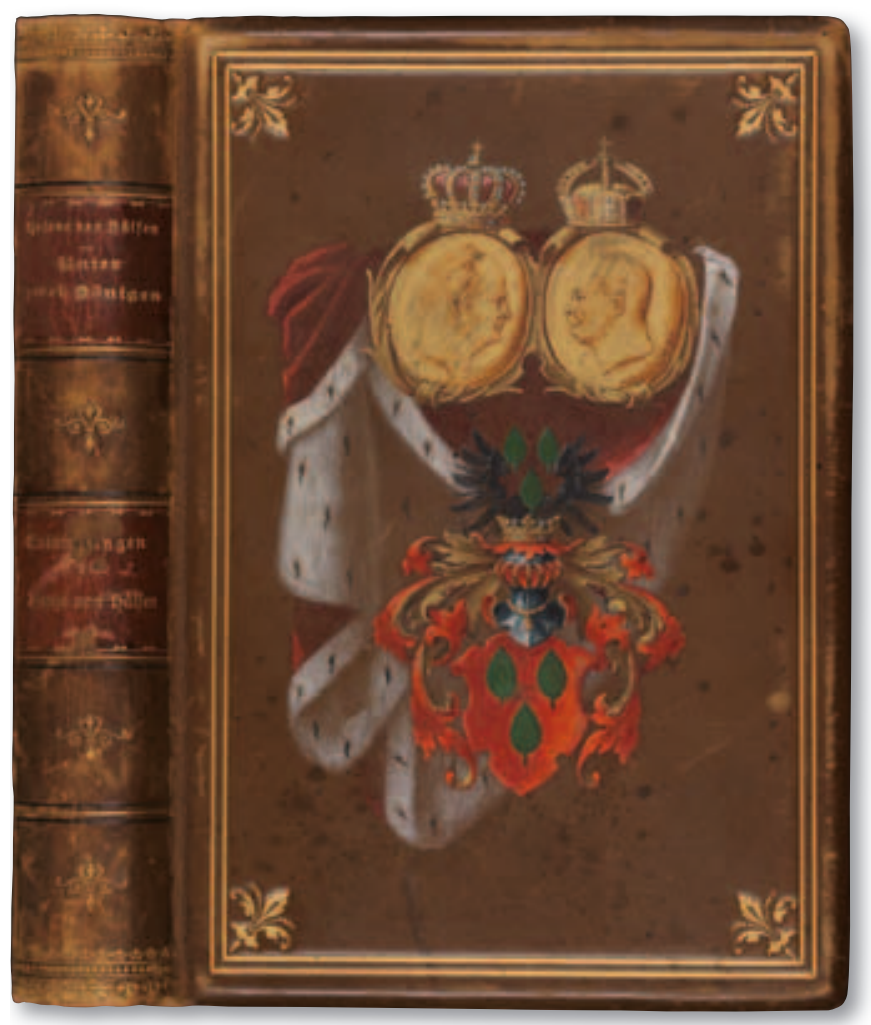

62/90004 / ES 44 